\title{
Yohimbine improves lipid and carbohydrate profiles without reduction in body weight in obese leptin-deficient ob/ob mice
}

\author{
Magdalena Kotańska', Monika Marcinkowska², Joanna Knutelska ${ }^{3}$, Małgorzata Zygmunt ${ }^{3}$, \\ Jacek Sapa ${ }^{3}$ \\ ${ }^{1}$ Department of Pharmacodynamics, Jagiellonian University Collegium Medicum, Kraków, Poland \\ 2 Department of Medicinal Chemistry, Jagiellonian University Collegium Medicum, Kraków, Poland \\ ${ }^{3}$ Department of Pharmacological Screening, Jagiellonian University Collegium Medicum Kraków, Poland
}

Kotańska M, Marcinkowska M, Knutelska J, Zygmunt M, Sapa J. Yohimbine improves lipid and carbohydrate profiles without reduction in body weight in obese leptin-deficient ob/ob mice. J Pre-Clin Clin Res. 2018; 12(2): 67-71. doi: 10.26444/jpccr/91404

\begin{abstract}
Introduction. Obesity, hyperglycaemia, hyperlipidemia and hypertension are the hallmarks of metabolic syndrome. The epidemic of obesity has been accompanied by an analogous rise in the number of patients with metabolic syndrome. Thus, obesity and related metabolic disturbances are the main objective of public health throughout the world. Previously, we have shown that yohimbine, a non-selective a2-adrenergic receptor antagonist, reduces body weight and improves disrupted lipid and carbohydrates profiles in rats with diet-induced obesity.

Material and method. In the presented study we have determined the effect of yohimbine on the body weight and carbohydrate and lipids levels in genetically obese leptin-deficient ob/ob mice and in lean C57BL6 mice.

Results. Yohimbine had a favourable effect on elevated levels of triglycerides, cholesterol and glucose. However, it did not have any influence on body weight.

Conclusions. The obtained data show that the weight reducing activity of yohimbine is primarily mediated via the interaction with a2-adrenergic receptor, and the subsequent enhanced release of noradrenaline which stimulates the $\beta 3$-adrenergic receptor presented on adipocytes. The favourable influence of yohimbie on impaired lipid-carbohydrate homeostasis is achieved via the blockade of a1-adrenergic receptor. We believe that non-selective a-adrenoceptor antagonists might offer therapeutic benefits in the treatment of obesity and metabolic disturbances.
\end{abstract}

\section{Key words}

obesity, body weight, yohimbine, a2-adrenoceptor, ob/ob mice

\section{INTRODUCTION}

According to the World Health Organization (WHO), approximately one billion people worldwide are overweight, and among them more than 300 million are clinically obese [1]. Not surprisingly, the WHO has defined obesity as an epidemic of the 21st century. Obesity along with dyslipidaemia, hyperglycaemia and hypertension, is one of the main clusters of the metabolic syndrome. In recent years, the increasing prevalence of obesity has been accompanied by an analogous rise in the number of patients with metabolic syndrome [2]. Both conditions constitute a serious therapeutic challenge and place a heavy burden on the health care system. Moreover, long-lasting, untreated metabolic disorders have been associated with increased mortality [3]. This shows the importance of an urgent development for effective and safe therapies.

Leptin is a protein secreted by the adipose tissue which mediate an anorectic effect and leads to increased energy expenditure. It inhibits the biosynthesis of triacylglycerol in the liver, adipose tissue and skeletal muscle, resulting in a decreased amount of lipids in the cells. Moreover, leptin increases tissue sensitivity to insulin and thus improves

Adress for correspondence: Magdalena Kotańska, Department of Pharmacodynamics, Jagiellonian University, Collegium Medicum, 9 Medyczna Street, 30-688 Kraków, Poland

E-mail:magda.dudek@uj.edu.pl

Received:10 April 2018; accepted: 21 May 2018 glucose tolerance [4]. The ob/ob mice carry mutations that prevent the synthesis of leptin, and thus suffer from leptin deficiency and, as a result, are obese. Additionally, ob/ob mice possess several characteristics that mimic metabolic syndrome in humans such as: hyperglycaemia, elevated plasma cholesterol levels or hypertriglyceridaemia [5]. For this reason, ob/ob mice have been frequently used as an animal model of obesity and obesity-related metabolic disturbances [6].

Much literature data has suggested that drugs such as sibutramine, bromocriptine or bupropion, apart from having appetite suppressing properties, are effective in reducing body weight due to their influence on $\alpha_{2}$-adrenoreceptors $[7,8,9]$. The $a$-adrenoreceptors are widely distributed in the body and are involved in the control of the function of adipocytes and pancreatic islet $\beta$-cells, as well as blood pressure [10]. The interaction with $\alpha_{2}$-adrenoceptors can lead to areduction of body weight indirectly via the induction of lipolysis and thermogenesis, while the blockade of $\alpha_{1}-$ adrenoceptors can improve disrupted lipid and carbohydrate profiles [11]. Several components of the metabolic syndrome, such as: obesity, imbalanced lipid-carbohydrate homeostasis or hypertension, are associated with over-activation of the sympathetic nervous system [12]. For this reason, molecules which block $\alpha$-adrenoceptors and can favourably modulate metabolic changes have attracted the interest of researchers.

Yohimbine is an indole based alkaloid isolated from the African tree Pausinystalia Yohimbe. It displays a wide 
range of pharmacological activities, such as: hypotensive, antipsychotic or reducing body weight [13]. Additionally, yohimbine has been studied as a potential treatment of xerostomia and erectile dysfunction. Yohimbine is a nonselective, potent antagonist of $\alpha_{2}$-adrenoreceptors. It also weakly interacts with the $\alpha_{1}$-adrenoreceptor type [11].

\section{OBJECTIVES}

Previously, we have found that yohimbine exerts an anorectic effect and consequently caused body weight reduction in an animal model of obesity induced by a high-fat diet. Additionally, yohimbine reduced the high glucose and triglyceride levels in the blood, which is believed to be mediated via the interaction with adrenergic receptors. As a continuation to our earlier experiments, in the presented study we attempted to assess the effect of a chronic administration of yohimbine on body weight in genetically obese leptin-deficient ob/ob mice, as well as to determine yohimbine's influence on lipid and carbohydrate profiles. The effects of yohimbine in ob/ob animals were determined in parallel on C57BL6 mice.

\section{MATERIALS AND METHOD}

Determination of the effect of yohimbine on body weight in the ob/ob mice and C57BL6 mice. Six-week-old male C57BL6 mice were divided into 3 groups of 6 animals: a control group receiving intraperitoneal water $(0.2 \mathrm{ml})$ and a group receiving intraperitoneal doses of yohimbine ( 2 or $5 \mathrm{mg} / \mathrm{kg}$ ) once per day in the morning between 09:00 - 10:00 0er a 30-day period. C57BL6 mice received a standard feed (Labofeed, Morawski Feed record label, Poland) with water available ad libitum.

Six-week-old male ob/ob mice (Jackson Laboratory, USA) were divided into 2 groups of 6 animals: a control group receiving intraperitoneal water $(0.2 \mathrm{ml})$ and a group receiving intraperitoneal doses of yohimbine $(5 \mathrm{mg} / \mathrm{kg})$ once per day in the morning between 09:00 -10:00 over a 30-day period. ob/ob Mice received feed as recommended by their breeder: JL Mouse Breeder Auto/no Hysil Labdiet (PMI NUTRITION INTL., LLC., England) with water available ad libitum. Intakes of food and water, and body weight were measured daily immediately prior to administration of drugs.

11 mice survived until the end of the experiment.

Biochemical analysis. On the 30th day of treatment, food was discontinued and on the 31st day plasma was collected from the animals from the carotid artery, 20 minutes after the intraperitoneal administration of heparin $500 \mathrm{j} / \mathrm{animal}$ and thiopental (70 $\mathrm{mg} / \mathrm{kg}$ b.w.). Each sample was then frozen in liquid nitrogen before storage at $-80^{\circ} \mathrm{C}$. Lipid profiles and glucose levels were determined. Standardized tests purchased from the Biomaxima Company S.A. (Poland) were used.

Statistical analysis. All statistical calculations were carried out with GraphPad Prism 6. Results were described as arithmetic means with standard errors of this mean (SEM). The statistical significance was calculated using a one-way analysis of variance (ANOVA) post-hoc Dunnett's Multiple Comparison Test or two-way ANOVA post-hoc Bonferroni or Student's t-test. Differences were considered statistically significant at $\mathrm{p} \leq 0.05:{ }^{\star} \mathrm{p} \leq 0.05,{ }^{* *} \mathrm{p} \leq 0.01,{ }^{* *} \mathrm{p} \leq 0.001$.

\section{RESULTS}

Effects of yohimbine on body weight, level of food and water intake in C57BL6 mice treated for 30 days with yohimbine. The C57BL6 mice treated for 30 days with yohimbine at $5 \mathrm{mg} /$ $\mathrm{kg}$ b.w. had a statistically significantly lower body weight on day 30 compared to the control mice group. They gained significantly less weight than control group mice (two-way ANOVA). There were no significant differences in weight on the first and last day of treatment (one-way ANOVA). The control mice significantly increased body weight during the experiment (one-way ANOVA). Changes in body weight over the 30 days of the treatment are shown in Figure 1A.

The C57BL6 mice receiving intraperitoneal yohimbine did not consume less food and water compared to the control mice. Therefore, it can be concluded that weight loss was not the result of any anorexic impact. The amount of food and water consumed is presented in Figure 2.

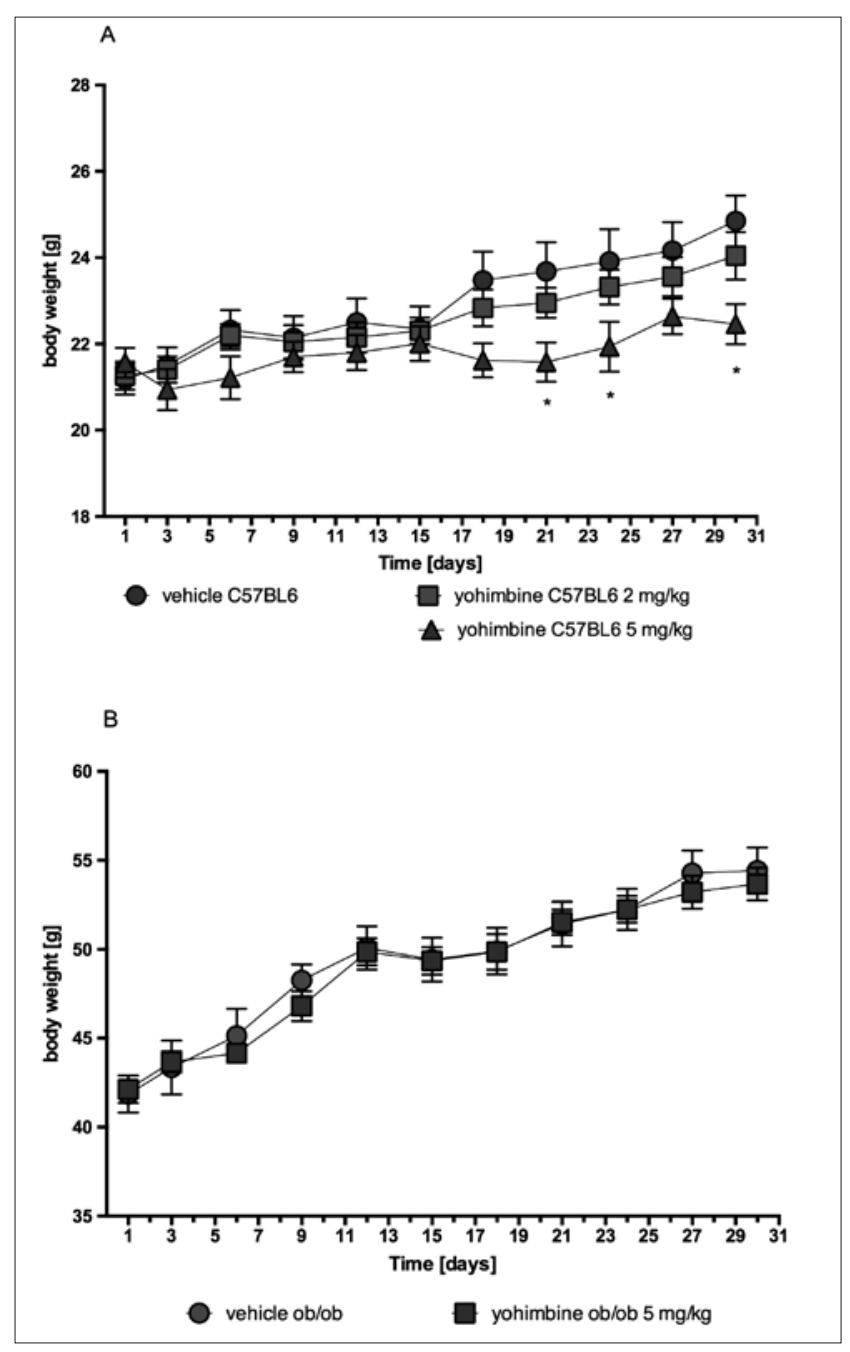

Figure 1. Weight change and food and water intakes during the treatment of ob/ ob mice or C57BL6 mice with yohimbin.e

A: Weight change in C57BL6 mice treated for 30 days with the test compound: yohimbine at a dose of $2 \mathrm{mg} / \mathrm{kg}$ b.w., i.p.; yohimbine at a dose of $5 \mathrm{mg} / \mathrm{kg}$ b.w., i.p. or vehicle - water; Means \pm SEM, $n=6$; Significant to control group (vehicle C57BL6): ${ }^{*} \mathrm{p}<0.05$ (two-way ANOVA); B: Weight change in ob/ob mice treated for 30 days with yohimbine at a dose of $5 \mathrm{mg} / \mathrm{kg}$ b.w., i.p. or vehicle - water; Means $\pm S E M, n=6$ 

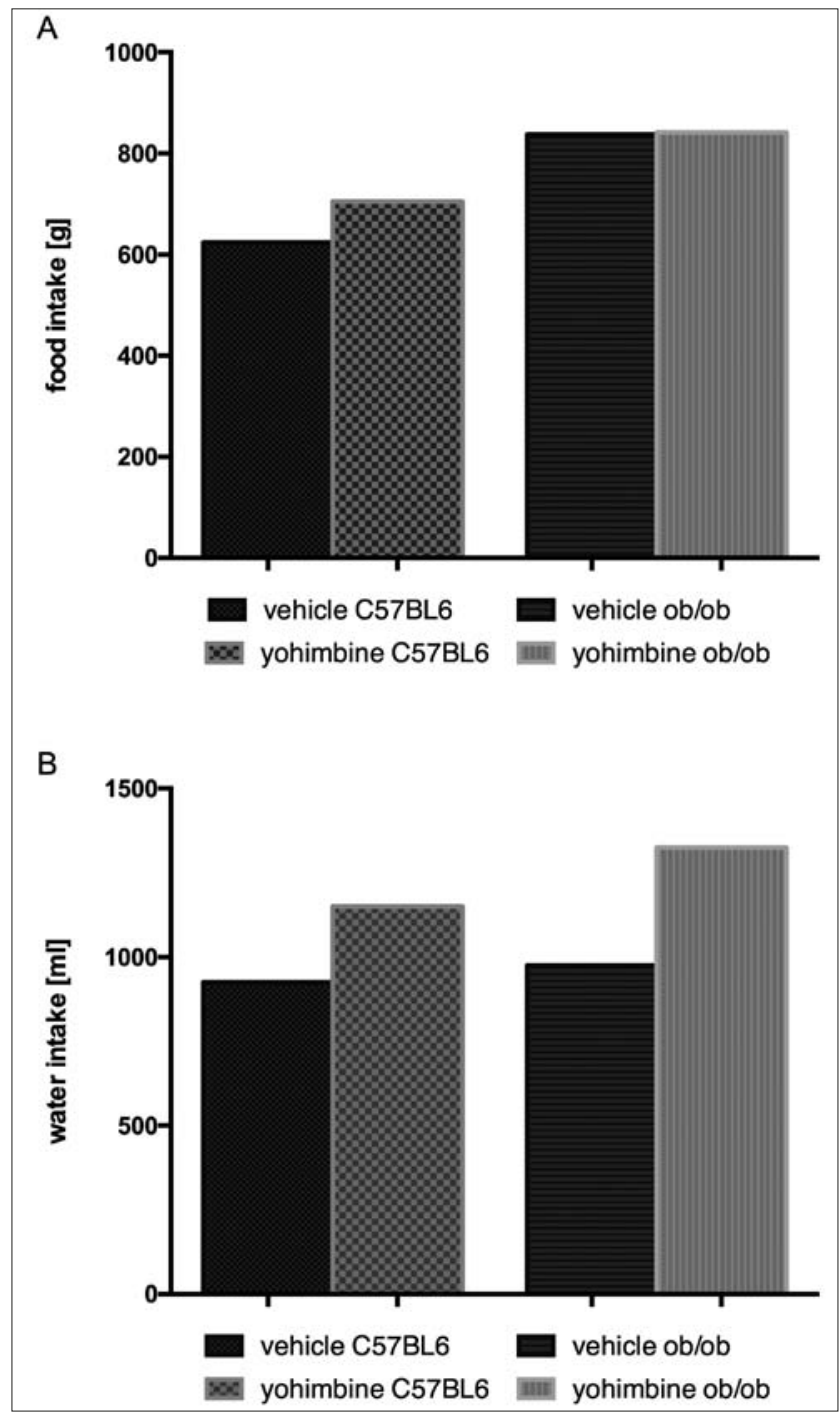

Figure 2. Food and water intakes during the treatment of ob/ob mice or C57BL6 mice with yohimbine.

Food and water intakes for 6 mice over 30 day treatment with test compound yohimbine at a dose of $5 \mathrm{mg} / \mathrm{kg}$ b.w., i.p. in C57BL6 or ob/ob mice

Effects of yohimbine on body weight, level of food and water intakes and lipid and carbohydrate profiles in ob/ ob mice treated for $\mathbf{3 0}$ days with yohimbine. Yohimbine used at a dose of $5 \mathrm{mg} / \mathrm{kg}$ b.w. for 30 days had no statistically significant effect on the body weight of ob/ob mice. There were no statistically significant differences between the control and treated groups (two-way ANOVA). Both groups had significantly increased their weight (one-way ANOVA, $\left.\mathrm{p}<0.001^{* * *}\right)$. Changes in body weight over the thirty days of treatment are shown in Figure 1B. The amount of ingested food and water is presented in Figure 2. Yohimbine administered for 30 days in ob/ob mice significantly reduced glucose levels and favorably affected the lipid profile (Student's t-test). Results are shown in Figure 3.

\section{DISCUSSION}

Obesity is a key component of metabolic syndrome and an established risk factor for cardiovascular diseases. Obesityrelated cardiac risk factors, such as hyperinsulinaemia, hyperlipidaemia, hypertension, generalized inflammation
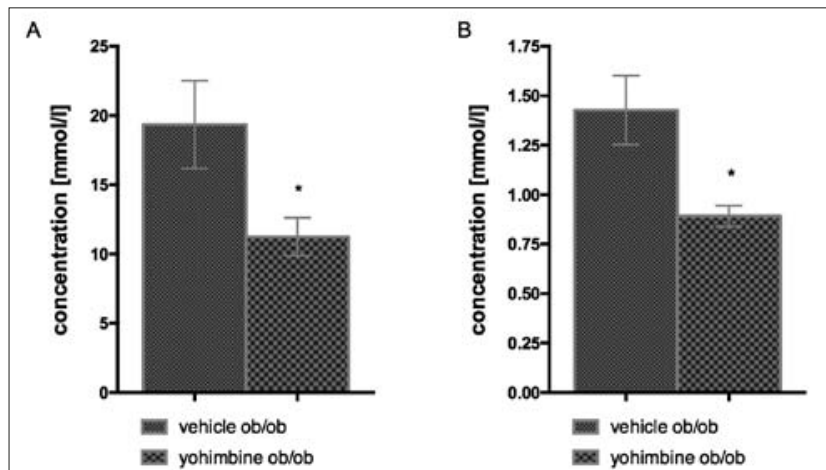

C
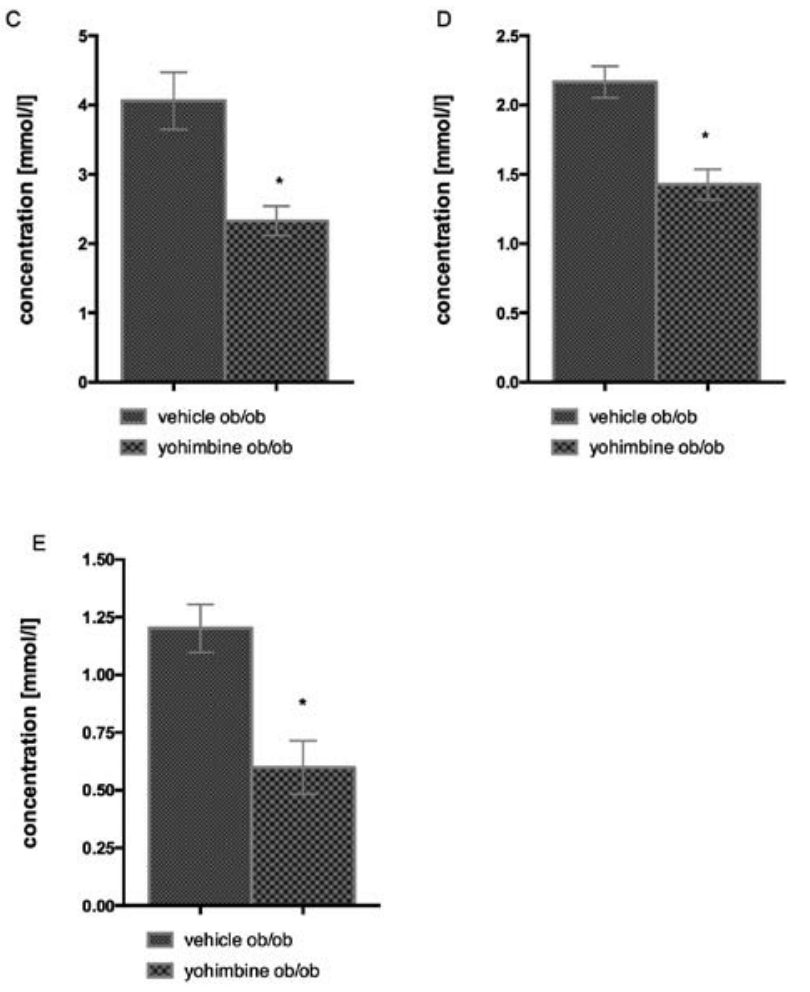

Figure 3. Effects of yohimbine on biochemical parameters after administration of yohimbine to ob/ob mice.

Level of: A: Glucose, B: TG, C: Cholesterol, D: HDL, E: LDL. Means \pm SEM, $n=6$; Significant to control ob/ob group - vehicle ob/ob * $p<0.05$ (Student's $t$-test).

and clotting abnormalities, are the targets for the treatment of obese patients who have not yet developed metabolic syndrome.. When untreated, these conditions can, in turn, lead to life-threatening heart disease [14].

The presented study was conducted to determine the effect of yohimbine on body weight and food intake in obese leptindeficient ob/ob mice and in their lean littermates: C57BL6 mice. The study began from the determination of an effective dosage of yohimbine, during which a significant reduction of body weight in the C57BL6 mice could be observed For this purpose, $2 \mathrm{mg} / \mathrm{kg}$ and $5 \mathrm{mg} / \mathrm{kg}$ of yohimbine was administrated for 30 days to C57BL6 animals. It was observedthat $5 \mathrm{mg} / \mathrm{kg}$ of yohimbine caused a significant reduction in body weight in C57BL6 mice. However, the amount of consumed food and water was not lower in comparison to the control group. Thus, It can conclude that the weight reduction activity was not a result of anorectic effect.

Chronic administration of $5 \mathrm{mg} / \mathrm{kg}$ of yohimbine was next administered to obese leptin-deficient ob/ob mice to see if the 
yohimbine influenced any body weight changes. According to previous literature reports, higher dosages of yohimbine are not recommended as yohimbine might undesirably affect blood pressure and heart rate, due to interaction with the central and peripheral adrenoceptors [11]. Nevertheless, yohimbine administrated for 30 days at dosage of $5 \mathrm{mg} / \mathrm{kg}$, did not have a statistically significant effect on body weight or food and water intake in mice with genetically-induced obesity (ob/ob mice).

$\mathrm{Ob} / \mathrm{ob}$ mice suffered from elevated noradrenaline levels in the ventromedial hypothalamic nuclei, a region in the brain which controls the peripheral lipid and glucose metabolism [15]. Additionally, the ventromedial hypothalamic nuclei are the main site of leptin activity. In the absence of leptin, as seen in ob/ob mice, some adrenergic receptor changes were observed. For instance, it has been stated that a decreased density of $a_{2}$-adrenoreceptors in ob/ob mice leads to an increased release of noradrenaline, that potentiate the obesehyperglycemic state. Noradrenaline triggers hyperglycaemia and impaired lipid profiles in these animals through the stimulation of $\alpha_{1}$-adrenoceptors [15]. Additionally, much literature data showed that $\beta_{3}$-adrenoceptor mRNA levels were considerably lower in white and brown adipose tissue of ob/ob mice, compared to lean mice [16]. $\beta_{3}$ adrenoceptors mediate lipolysis in white adipose tissue [17], while in brown adipose tissue $\beta_{3}$-adrenoceptors trigger thermogenesis. Both effects contribute to the reduction of body weight [18].

The primary mechanism of the action of yohimbine is related with the blockade of presynaptic $\alpha_{2}$-adrenoreceptors which enhances the release of noradrenaline into the synaptic cleft. Noradrenaline, in turn, stimulates the $\beta_{3}$-adrenoceptor, which undoubtedly results in the body weight reduction. This effect has been confirmed in the animal model of obesity induced by a high-fat diet [11]. Similarly, it can be assumed that the weight-reducing activity of yohimbine in the lean littermates C57BL6 mice is the result of interaction with $\alpha_{2}$-adrenoreceptors. However, the lack of any effect in reducing body weight after administration of yohimbine in genetically altered animals, might be related with the inability to interact with both $\alpha_{2}$ - and $\beta_{3}$-adrenoceptors, as their function are impaired in ob/ob mice. Thus, the obtained results in the current study concur with the authors' previous findings that the effect of yohimbine in reducing body weight is mainly mediated via the blockade of $\alpha_{2}$-adrenoceptors, and consequently with the indirect stimulation of $\beta_{3}$ adrenoceptors by the released noradrenaline.

The presented study additionally determined the effect of yohimbine on the lipid and glucose profile. In contrast to the lack of weight reducing effects, a significant decrease was observed in the glucose level and improved lipid profiles in ob/ob mice, by means of a significant reduction of triglyceride, total cholesterol and low-density lipoprotein (LDL) levels. Similar results were observed in the authors' previous studies which showed that yohimbine favourably moderates lipid and glucose profiles in obese rats, induced by a high-fat feed [11]. The influence on lipid profile is most likely related with the blocking effect on $\alpha_{1}$-adrenoceptor [19], as yohimbine interacts additionally with this type of adrenergic receptors. The $\alpha_{1}$-adrenoceptor blockade causes an enhancement of noradrenaline levels, which increases glucose entrance into adipocytes and consequently decreases the amount of glucose in the blood.
The current results show that yohimbine can favourably influence the imbalanced lipid-carbohydrate homeostasis in the presence of leptin deficiency and impaired function of $\alpha_{2}-$ and $\beta_{3}$-adrenoceptors. Many reports have shown an increasing number of patients suffering from leptin resistance constitute a serious therapeutic challenge. Therefore, the present results highlight the great potential of $\alpha_{1}$-adrenoceptor antagonists in the future treatment of disrupted lipid and carbohydrate profiles, particularly in patients with metabolic disorders and leptin resistance. Furthermore, yohimbine is frequently used in the treatment of erectile dysfunction in men. Some of these patients may suffer from disrupted lipid-carbohydrate homeostasis. Patients who have already implemented the treatment with yohimbine may additionally benefit from its favourable effects on the disrupted lipid and glucose profiles.

The auithors believe that $\alpha$-adrenoceptor are an interesting therapeutic target and should be considered as a potential treatment for metabolic disturbances. Several components of the metabolic syndrome are related to an enhanced stimulation of the sympathetic nervous system [12]. Moreover, many undesirable reactions are mediated via the activation of a-adrenoceptors, and in particular enhanced the release of free fatty acids and glucose. Thus, targeting the over-activated sympathetic nervous system with adrenoceptor antagonists, appears to be a judicious and suitable therapeutic approach for the treatment of metabolic disturbances [20].

The authors recognized the following limitation of the current study: that although insulin levels were not determined during the experiments, such analysis might be of interest as $\alpha_{2}$-adrenoceptor antagonists can positively modulate the pancreatic function and control the secretion of insulin secretion. This issue will be considered in the authors' future studies.

\section{CONCLUSIONS}

The study shows that $\alpha_{2}$-adrenoceptor antagonist yohimbine, reduced body weight in the C57BL6 mice, but not in obese leptin-deficient ob/ob mice. The $\alpha_{2}$-adrenoceptor antagonists unblocked the release of noradrenaline, which may increase the stimulation of $\beta_{3}$-adrenoceptors and cause body weight reduction. In the absence of leptin, and the impaired function of $\alpha_{2}$ and $\beta_{3}$-adrenoceptors, as seen in ob/ob mice, yohimbine is ineffective to provide weight reducing activity. However, yohimbine improved lipid and carbohydrate profiles in these animals, which is most likely related with the blockade of the $\alpha_{1}$-adrenoceptor, since yohimbine interacts additionally with $a_{1}$-adrenoceptors in high dose. The authors believe that nonselective $\alpha$-adrenoceptor antagonists might offer therapeutic benefits in the treatment of disrupted lipid-carbohydrate homeostasis.

\section{REFERENCES}

1. World Health Organization Obesity and Overweight: World Health Organization global strategy on diet, physical activity and health fact sheet. [Accessed September 16, 2005]. 2003.

2. Wyatt SB, Winters KP, Dubbert PM (2006) Overweight and obesity: prevalence, consequences, and causes of a growing public health problem. Am J Med Sci 331(4): 166-74

3. Ginsberg HN, MacCallum PR (2009) The Obesity, Metabolic Syndrome, and Type 2 Diabetes Mellitus Pandemic: Part I. Increased Cardiovascular Disease Risk and the Importance of Atherogenic Dyslipidemia in 
Persons With the Metabolic Syndrome and Type 2 Diabetes Mellitus. J Cardiometab Syndr 4(2): 113-119

4. Gogga P, Karbowska J, Meissner W, Kochan Z (2011) Role of leptin in the regulation of lipid and carbohydrate metabolism. Postepy Hig. Med. Dosw. (online) 65: 255-262

5. Kennedy AJ, Ellacott KLJ, King VL, Hasty AH (2010) Mouse models of the metabolic syndrome. Dis Model Mech 3(3-4): 156-166

6. Ohyama T, Sato K, Yamazaki Y, Hashizume H, Horiguchi N, Kakizaki S, Mori M, Kusano M, Yamada M (2014) MK-0626, a selective DPP4 inhibitor, attenuates hepatic steatosis in ob/ob mice. World J Gastroenterol 20: 16227-35

7. Janhunen SK, van der Zwaal EM, la Fleur SE, Adan RAH (2011) Inverse agonism at $\alpha 2 \mathrm{~A}$ adrenoreceptors augments the hypophagic effect of sibutramine in rats. Obesity 19: 1979-1986

8. Mukherjee R, Yun JW (2013) Bromocriptine inhibits adipogenesis and lipogenesis by agonistic action on a2-adrenergic receptor in 3T3-L1 adipocyte cells. Mol Biol Rep 40: 3783-3792

9. Janhunen SK, la Fleur SE, Adan RA (2013) Blocking alpha2A adrenoreceptors, but not dopamine receptors, augments bupropioninduced hypophagia in rats. Obesity 21: 700-8

10. Philipp M, Brede M, Hein L (2002) Physiological significance of a2adrenergic receptor subtype diversity: one receptor is not enough. Am J Physiol Regul Integr Comp Physiol 283: 287-295

11. Dudek M, Knutelska J, Bednarski M, Nowiński L, Zygmunt M, Mordyl B, Głuch-Lutwin M, Kazek G, Sapa J, Pytka K (2015) The comparison of the anorectic effect and safety of alpha $a_{2}$-adrenoceptor ligands: guanfacine and yohimbine in rats with diet-induced obesity. PlosOne DOI:10.1371/journal.pone.0141327
12. dos Santos Moreira MC, de Jesej Pinto IS, Fajemiroye JO, Colombari E, da Silva Reis AA, Freiria-Oliveira AH, Ferreira-Neto ML, Pedrino GR. Does the sympathetic nervous system contribute to the pathophysiology of metabolic syndrome? (2015) Front Physiol 6: 234

13. Beille PE (2013) Scientific Opinion on the evaluation of the safety in use of Yohimbe (Pausinystalia yohimbe) EFSA J 11(7): 3302

14. Ades PA, Savage PD, Toth MJ, Schneider DJ, Audelin MC, Bunn JY, Ludlow M (2008) The influence of obesity and consequent insulin resistance on coronary risk factors in medically treated patients with coronary disease. Int J Obes 32: 967-974

15. Boundy VA, Cincotta AH (2000) Hypothalamic adrenergic receptor changes in the metabolic syndrome of genetically obese $(o b / o b)$ mice. Am J Physiol Regul Integr Comp Physiol 279(2): R505-514

16. Evans BA, Papaioannou M, Anastasopoulos F, Summers RJ (1998) Differential regulation of $\beta 3$-adrenoceptors in gut and adipose tissue of genetically obese (ob/ob) C57BL/6J-mice. Br J Pharmacol 124: 763 - 771

17. Lafontan M, Berlan M (1993) Fat cell adrenergic receptors and the control of white and brown fat cell function. J Lipid Res 34: 1057-1091

18. Arch JR, Ainsworth AT, Cawthorne MA, Piercy V, Sennitt MV, Thody VE, Wilson C, Wilson S (1984) Atypical $\beta$-adrenoceptors on brown adipocytes as target for anti-obesity drugs. Nature 309: 163-165

19. Flechtner-Mors M, Jenkinson CP, Alt A, Biesalski HK, Adler G, Ditschneit HH (2004) Sympathetic Regulation of Glucose Uptake by the $\alpha_{1}$-Adrenoceptor in Human Obesity. Obesity 12(4): 612-620

20. Schlaich M, Straznicky N, Lambert E, Lambert G (2015) Metabolic syndrome: a sympathetic disease? Lancet Diabetes Endocrinol 3(2): $148-157$. 